\title{
Physics... in action
}

Dr. Vassilis Lembessis, The University of Southeastern Europe, Athens

The electric guitar is the one instrument that epitomises rock $I$ music. As with so many other ear-shattering inventions, physics played its part.

The birth of rock music in the mid-1950s was accompanied with a switch from acoustic to electric guitars. For an electric guitar, we know that we can make a metal string electric with the help

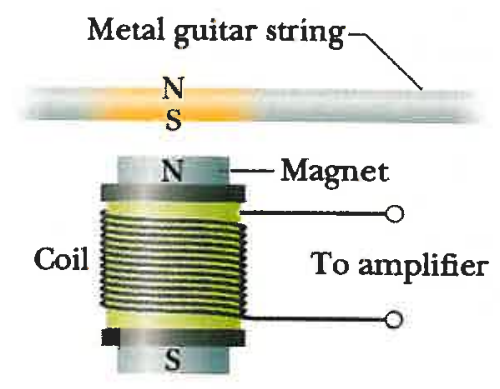

of a magnet. Under the strings we put a series of bar magnets known as the pickups (as shown in the figure). These magnets are placed inside a solenoid having one of their poles very close to the strings. The presence of the magnet produces a north and a south pole in the section of the metal string just above the magnet. When the guitarist plays a string it vibrates, changing the magnetic flux through the solenoid generating an induction current in the solenoid. This current goes to properly designed electronic devices for amplification and the generation of sound.

Thus the operation of the electric guitar is radically different from that of the acoustic. Whereas in the acoustic guitar the sound depends on the acoustic resonance produced in the hollow body of the instrument by the oscillations of the strings, the electric guitar is a solid instrument, so there is no resonance. Instead the oscillations of the metal strings are sensed by electric pickups that send signals to an amplifier and a set of speakers. As the string oscillates toward and away from the coil, the induced current changes direction at the same frequency as the string's oscillations, thus relaying the frequency of oscillation to the amplifier and speaker.

It was Jimmy Hendrix who first understood the electric guitar as an electronic instrument and who showed that we could gain further control over the music by changing the number of turns of the solenoid and thus the amount of electro magnetic flux induced in the coils and therefore their relative sensitivity to string vibrations.

\section{Further reading}

Halliday-Resnick-Walker: Fundamentals of Physics 6 th Edition, Wiley International. 